\title{
On the Solvability of Second-Order Impulsive Differential Equations with Antiperiodic Boundary Value Conditions
}

\author{
Yepeng Xing $^{1}$ and Valery Romanovski ${ }^{2}$ \\ ${ }^{1}$ Department of Applied Mathematics, Shanghai Normal University, Shanghai 200234, China \\ ${ }^{2}$ Center for Applied Mathematics and Theoretical Physics, University of Maribor, SI-2000 Maribor, Slovenia
}

Correspondence should be addressed to Yepeng Xing, ypxing@shnu.edu.cn

Received 3 July 2008; Accepted 10 November 2008

Recommended by Colin Rogers

We prove existence results for second-order impulsive differential equations with antiperiodic boundary value conditions in the presence of classical fixed point theorems. We also obtain the expression of Green's function of related linear operator in the space of piecewise continuous functions.

Copyright (C) 2008 Y. Xing and V. Romanovski. This is an open access article distributed under the Creative Commons Attribution License, which permits unrestricted use, distribution, and reproduction in any medium, provided the original work is properly cited.

\section{Introduction and preliminaries}

Many evolution processes are characterized by the fact that at certain moments of time they experience a change of state abruptly. Consequently, it is natural to assume that these perturbations act instantaneously, that is, in the form of impulses. It is known that many biological phenomena involving threshold, bursting rhythm models in medicine and biology, optimal control models in economics, pharmacokinetics, and frequency modulated systems do exhibit impulse effects. The branch of modern, applied analysis known as "impulsive" differential equations provides a natural framework to mathematically describe the aforementioned jumping processes. The reader is referred to monographs [1-4] and references therein for some nice examples and applications to the above areas.

In this paper, we mainly study the following second-order impulsive differential equations with antiperiodic boundary value conditions:

$$
\begin{gathered}
u^{\prime \prime}=f\left(t, u, u^{\prime}\right), \quad t \in[0, T] \backslash \Omega, \\
u\left(t_{k}^{+}\right)=u\left(t_{k}\right)+I_{k}\left(u\left(t_{k}\right)\right), \quad u^{\prime}\left(t_{k}^{+}\right)=u^{\prime}\left(t_{k}\right)+J_{k}\left(u\left(t_{k}\right)\right), \quad k=1,2, \ldots, m, \\
u(0)=-u(T), \quad u^{\prime}(0)=-u^{\prime}(T),
\end{gathered}
$$


where $\Omega:=\bigcup_{i=1}^{m} t_{i}$ and $f:[0, T] \times \mathbb{R}^{n} \times \mathbb{R}^{n} \rightarrow \mathbb{R}^{n}$ is continuous on $[0, T] \backslash \Omega \times \mathbb{R}^{n} \times \mathbb{R}^{n}$, $I, J: \mathbb{R}^{n} \rightarrow \mathbb{R}^{n}$ are continuous functions.

In [4-12], the authors studied the existence of antiperiodic solutions for first-order, second-order, or high-order differential equations without impulses, and in [3, 13-16] the authors were concerned with the antiperiodic solutions of first-order impulsive differential equations. Also we should mention the work by Cabada et al. in [17] which is concerned with a certain $n$th order linear differential equation with constant impulses at fixed times and nonhomogeneous periodic boundary conditions. So far, to the best of our knowledge, this is the first work to deal with the antiperiodic solutions to second-order differential equations with nonconstant impulses. Our method to prove the existence of antiperiodic solutions is based on the works in $[13,18,19]$. We should point out that it is Christopher C. Tisdell who started with this method.

The article is organized as follows. In Section 2, we present the expression of Green's functions of related linear operator in the space of piecewise continuous functions. Section 3 contains the main results of the paper and is devoted to the existence of solutions to (1.1). There, differential inequalities are developed and applied to prove the existence of at least one solution to (1.1). In Section 4, a couple of examples are given to illustrate how the main results work.

To understand the notation used above and the ideas in the remainder of the paper, we now briefly introduce some appropriate concepts connected with impulsive differential equations. Most of the following notation can be found in $[1,2,4,5]$. We assume that $f\left(t_{k}^{+}, x, y\right):=\lim _{t \rightarrow t_{k}^{+}} f(t, x, y), f\left(t_{k^{-}}^{-}, x, y\right):=\lim _{t \rightarrow t_{k}^{-}} f(t, x, y)$ exist and $f\left(t_{k^{-}}^{-}, x, y\right)=$ $f\left(t_{k}, x, y\right), k=1,2, \ldots, m$. We introduce and denote the Banach space $P C\left([0, T], \mathbb{R}^{n}\right)$ by

$$
\begin{aligned}
P C\left([0, T] ; \mathbb{R}^{n}\right):= & \left\{u:[0, T] \longrightarrow \mathbb{R}^{n}, u \in C\left([0, T] \backslash \Omega, \mathbb{R}^{n}\right),\right. \\
& \left.u \text { is left continuous at } t=t_{k}, \text { the right-hand limit } u\left(t_{k}^{+}\right) \text {exists }\right\}
\end{aligned}
$$

with the norm $\|u\|_{P C}:=\sup _{t \in[0, T]}\|u(t)\|$, where $\|\cdot\|$ is the usual Euclidean norm and $\langle\cdot, \cdot\rangle$ will be the Euclidean inner product.

In a similar fashion to the above, define and denote the Banach space $P C^{1}\left([0, T], \mathbb{R}^{n}\right)$ by

$$
\begin{aligned}
P C^{1}\left([0, T] ; \mathbb{R}^{n}\right):= & \left\{u \in P C\left([0, T] ; \mathbb{R}^{n}\right): u \in C^{1}\left([0, T] \backslash \Omega, \mathbb{R}^{n}\right),\right. \\
& \text { the limits } \left.u^{\prime}\left(t_{k}^{-}\right), u^{\prime}\left(t_{k}^{+}\right) \text {exist with } u^{\prime}\left(t_{k}^{-}\right)=u^{\prime}\left(t_{k}\right)\right\}
\end{aligned}
$$

with the norm $\|u\|_{P C^{1}}:=\sup _{t \in[0, T]}\left\{\|u(t)\|_{P C},\left\|u^{\prime}(t)\right\|_{P C}\right\}$.

The following fixed point theorem is our main tool to prove the existence of at least one solution to (1.1).

Schaefer's fixed point theorem [19]

Let $X$ be a Banach space and let $A: X \rightarrow X$ be a completely continuous operator. Then, either

(i) the operator equation $x=\lambda A x$ has a solution for $\lambda=1$, or

(ii) the set $S:=\{x \in X, x=\lambda A x, \lambda \in] 0,1[\}$ is unbounded. 


\section{Expression of Green's function}

In this part, we present the expression of Green's functions for second order impulsive equations with antiperiodic conditions.

Lemma 2.1. Assume $p \geq 0$ and $q>0$ are two constants. Let $\alpha=\left(p+\sqrt{p^{2}+4 q}\right) / 2, \beta=(p-$ $\left.\sqrt{p^{2}+4 q}\right) / 2$. Then for any $h(t) \in P C\left([0, T], \mathbb{R}^{n}\right), u(t)$ solves

$$
\begin{gathered}
u^{\prime \prime}-p u^{\prime}-q u=h(t), \quad t \in[0, T], t \neq t_{k}, k=1,2, \ldots, m, \\
u\left(t_{k}^{+}\right)=u\left(t_{k}\right)+I_{k}\left(u\left(t_{k}\right)\right), \quad u^{\prime}\left(t_{k}^{+}\right)=u^{\prime}\left(t_{k}\right)+J_{k}\left(u\left(t_{k}\right)\right), \quad k=1,2, \ldots, m, \\
u(0)=-u(T), \quad u^{\prime}(0)=-u^{\prime}(T)
\end{gathered}
$$

if and only if $u(t)$ is the solution of integral equation

$$
u(t)=\int_{0}^{T} G(t, s) h(s) d s+\sum_{i=1}^{m} H\left(t, t_{i}\right) I_{i}\left(u\left(t_{i}\right)\right)+\sum_{i=1}^{m} G\left(t, t_{i}\right) J_{i}\left(u\left(t_{i}\right)\right)
$$

where

$$
\begin{gathered}
G(t, s)=\frac{1}{\alpha-\beta} \begin{cases}-\frac{e^{\alpha(T+t-s)}}{1+e^{\alpha T}}+\frac{e^{\beta(T+t-s)}}{1+e^{\beta T}}, & 0 \leq t \leq s \leq T, \\
\frac{e^{\alpha(t-s)}}{1+e^{\alpha T}}-\frac{e^{\beta(t-s)}}{1+e^{\beta T}}, & 0 \leq s<t \leq T,\end{cases} \\
H(t, s)=\frac{1}{\alpha-\beta} \begin{cases}\frac{\beta e^{\alpha(T+t-s)}}{1+e^{\alpha T}}-\frac{\alpha e^{\beta(T+t-s)}}{1+e^{\beta T}}, & 0 \leq t \leq s \leq T, \\
-\frac{\beta e^{\alpha(t-s)}}{1+e^{\alpha T}}+\frac{\alpha e^{\beta(t-s)}}{1+e^{\beta T}}, & 0 \leq s<t \leq T .\end{cases}
\end{gathered}
$$

Proof. Assume $u(t)$ is a solution of (2.1) and let $v(t)=u^{\prime}(t)-\beta u(t)$ for $t \neq t_{k}, k=1,2, \ldots, m$. We have

$$
v^{\prime}(t)-\alpha v(t)=u^{\prime \prime}(t)-(\alpha+\beta) u^{\prime}(t)+\alpha \beta u(t)=u^{\prime \prime}(t)-p u^{\prime}(t)-q u(t)=h(t) .
$$

Then for $t \in\left[0, t_{1}\right]$,

$$
v(t)=e^{\alpha t}\left[e^{0} v(0)+\int_{0}^{t} e^{-\alpha s} h(s) d s\right]=e^{\alpha t}\left[v(0)+\int_{0}^{t} e^{-\alpha s} h(s) d s\right] .
$$


This implies $v\left(t_{1}\right)=e^{\alpha t_{1}}\left[v(0)+\int_{0}^{t_{1}} e^{-\alpha s} h(s) d s\right]$. Consequently, from the impulsive condition in (2.1) we get that

$$
v\left(t_{1}^{+}\right)=v\left(t_{1}\right)+J_{1}\left(u\left(t_{1}\right)\right)-\beta I_{1}\left(u\left(t_{1}\right)\right)=e^{\alpha t_{1}}\left(v(0)+\int_{0}^{t_{1}} e^{-\alpha s} h(s) d s\right)+\Delta_{1}
$$

where $\Delta_{i}:=J_{i}\left(u\left(t_{i}\right)\right)-\beta I_{i}\left(u\left(t_{i}\right)\right), i=1,2, \ldots, m$. Now we integrate (2.3) from $t_{1}$ to $t \in\left(t_{1}, t_{2}\right]$ and use (2.5) to obtain

$$
v(t)=e^{\alpha t}\left[e^{-\alpha t_{1}} v\left(t_{1}^{+}\right)+\int_{t_{1}}^{t} e^{-\alpha s} h(s) d s\right]=e^{\alpha t} v(0)+e^{\alpha\left(t-t_{1}\right)} \Delta_{1}+\int_{0}^{t} e^{\alpha(t-s)} h(s) d s
$$

It follows that

$$
v\left(t_{2}^{+}\right)=v\left(t_{2}\right)+\Delta_{2}=e^{\alpha t_{2}} v(0)+e^{\alpha\left(t_{2}-t_{1}\right)} \Delta_{1}+\int_{0}^{t_{2}} e^{\alpha\left(t_{2}-s\right)} h(s) d s+\Delta_{2}
$$

Similarly, we have for $t \in\left(t_{2}, t_{3}\right]$ that

$$
v(t)=e^{\alpha t} v(0)+e^{\alpha\left(t-t_{1}\right)} \Delta_{1}+e^{\alpha\left(t-t_{2}\right)} \Delta_{2}+\int_{0}^{t} e^{\alpha(t-s)} h(s) d s .
$$

To sum up, we have for $t \in[0, T]$ that

$$
v(t)=e^{\alpha t} v(0)+\sum_{t_{i} \in[0, t)} e^{\alpha\left(t-t_{i}\right)} \Delta_{i}+\int_{0}^{t} e^{\alpha(t-s)} h(s) d s .
$$

Since $v(t)=u^{\prime}(t)-\beta u(t)$, we can deduce in a similar way as to deal with $h(t)=v^{\prime}(t)-\alpha v(t)$ to obtain

$$
u(t)=e^{\beta t} u(0)+\sum_{t_{i} \in[0, t)} e^{\beta\left(t-t_{i}\right)} I_{i}\left(u\left(t_{i}\right)\right)+\int_{0}^{t} e^{\beta(t-s)} v(s) d s .
$$

Now we are in position to show the expression of $u(t)$ for $t \in[0, T]$. To do that, we need to compute $\int_{0}^{t} e^{\beta(t-s)} v(s) d s$ in (2.10). In what follows we present the expression of $u(t)$ for $t \in\left(t_{1}, t_{2}\right],\left(t_{2}, t_{3}\right]$ step by step and then obtain the general form of $u(t)$ for $t \in[0, T]$. 
First of all, for $t \in\left(t_{1}, t_{2}\right]$, we have

$$
\int_{0}^{t} e^{\beta(t-s)} v(s) d s=\int_{0}^{t_{1}} e^{\beta(t-s)} v(s) d s+\int_{t_{1}}^{t} e^{\beta(t-s)} v(s) d s .
$$

See that

$$
\begin{aligned}
& \int_{0}^{t_{1}} e^{\beta(t-s)} v(s) d s=\int_{0}^{t_{1}} e^{\beta(t-s)}\left[e^{\alpha s} v(0)+\int_{0}^{s} e^{\alpha(s-\tau)} h(\tau) d \tau\right] d s, \\
& \int_{t_{1}}^{t} e^{\beta(t-s)} v(s) d s=\int_{t_{1}}^{t} e^{\beta(t-s)}\left[e^{\alpha s} v(0)+\Delta_{1} e^{\alpha\left(s-t_{1}\right)}+\int_{0}^{s} e^{\alpha(s-\tau)} h(\tau) d \tau\right] d s .
\end{aligned}
$$

\section{Consequently,}

$$
\int_{0}^{t} e^{\beta(t-s)} v(s) d s=\int_{0}^{t} e^{\beta(t-s)}\left[e^{\alpha s} v(0)+\int_{0}^{s} e^{\alpha(s-\tau)} h(\tau) d \tau\right] d s+\Delta_{1} \int_{t_{1}}^{t} e^{\beta(t-s)} e^{\alpha\left(s-t_{1}\right)} d s
$$

Integrate $\int_{0}^{t} e^{\beta(t-s)}\left[\int_{0}^{s} e^{\alpha(s-\tau)} h(\tau) d \tau\right] d s$ by parts to get

$$
\frac{e^{\beta t}}{\alpha-\beta} \int_{0}^{t} h(s) e^{-\alpha s}\left[e^{(\alpha-\beta) t}-e^{(\alpha-\beta) s}\right] d s .
$$

Thus,

$$
\begin{gathered}
\int_{0}^{t} e^{\beta(t-s)} v(s) d s=\frac{e^{\beta t}}{\alpha-\beta}\left\{v(0)\left[e^{(\alpha-\beta) t}-1\right]+e^{-\alpha t_{1}} \Delta_{1}\left[e^{(\alpha-\beta) t}-e^{(\alpha-\beta) t_{1}}\right]\right. \\
\left.+\int_{0}^{t} h(s) e^{-\alpha s}\left[e^{(\alpha-\beta) t}-e^{(\alpha-\beta) s}\right] d s\right\} .
\end{gathered}
$$

Similarly, we have for $t \in\left(t_{2}, t_{3}\right]$ that

$$
\begin{aligned}
\int_{0}^{t} e^{\beta(t-s)} v(s) d s=\frac{e^{\beta t}}{\alpha-\beta}\{ & v(0)\left[e^{(\alpha-\beta) t}-1\right]+e^{-\alpha t_{1}} \Delta_{1}\left[e^{(\alpha-\beta) t}-e^{(\alpha-\beta) t_{1}}\right] \\
& \left.+e^{-\alpha t_{2}} \Delta_{2}\left[e^{(\alpha-\beta) t}-e^{(\alpha-\beta) t_{2}}\right]+\int_{0}^{t} h(s) e^{-\alpha s}\left[e^{(\alpha-\beta) t}-e^{(\alpha-\beta) s}\right] d s\right\}
\end{aligned}
$$


Now we consider $u(t)$ for $t \in\left(t_{1}, t_{2}\right]$. Clearly,

$$
\begin{aligned}
u(t)= & e^{\beta t} u(0)+e^{\beta\left(t-t_{1}\right)} I_{1}\left(u\left(t_{1}\right)\right) \\
& +\frac{e^{\beta t}}{\alpha-\beta}\left\{v(0)\left[e^{(\alpha-\beta) t}-1\right]+e^{-\alpha t_{1}} \Delta_{1}\left[e^{(\alpha-\beta) t}-e^{(\alpha-\beta) t_{1}}\right]+\int_{0}^{t} h(s) e^{-\alpha s}\left[e^{(\alpha-\beta) t}-e^{(\alpha-\beta) s}\right] d s\right\} .
\end{aligned}
$$

Noting that $v(0)=u^{\prime}(0)-\beta u(0)$, we have

$$
\begin{aligned}
u(t)=\frac{1}{\alpha-\beta}\{( & \left.u^{\prime}(0)-\beta u(0)\right) e^{\alpha t}+\left(\alpha u(0)-u^{\prime}(0)\right) e^{\beta t} \\
& \left.+\int_{0}^{t} h(s)\left[e^{\alpha(t-s)}-e^{\beta(t-s)}\right]+e^{\alpha\left(t-t_{1}\right)} \Delta_{1}-e^{\beta\left(t-t_{1}\right)} \widetilde{\Delta}_{1}\right\},
\end{aligned}
$$

where $\widetilde{\Delta}_{i}$ is denoted by $\widetilde{\Delta}_{i}=J_{1}\left(u\left(t_{1}\right)\right)-\alpha I_{1}\left(u\left(t_{1}\right)\right), i=1,2, \ldots, m$. Similarly, for $t \in\left(t_{2}, t_{3}\right]$ there holds

$$
\begin{aligned}
u(t)=\frac{1}{\alpha-\beta}\{ & \left(u^{\prime}(0)-\beta u(0)\right) e^{\alpha t}+\left(\alpha u(0)-u^{\prime}(0)\right) e^{\beta t} \\
& \left.+\int_{0}^{t} h(s)\left[e^{\alpha(t-s)}-e^{\beta(t-s)}\right]+e^{\alpha\left(t-t_{1}\right)} \Delta_{1}+e^{\alpha\left(t-t_{2}\right)} \Delta_{2}-e^{\beta\left(t-t_{1}\right)} \tilde{\Delta}_{1}-e^{\beta\left(t-t_{2}\right)} \tilde{\Delta}_{2}\right\} .
\end{aligned}
$$

Thus, for $t \in[0, T]$,

$$
\begin{aligned}
u(t)=\frac{1}{\alpha-\beta}\{ & \left(u^{\prime}(0)-\beta u(0)\right) e^{\alpha t}+\left(\alpha u(0)-u^{\prime}(0)\right) e^{\beta t} \\
& \left.+\int_{0}^{t} h(s)\left[e^{\alpha(t-s)}-e^{\beta(t-s)}\right]+\sum_{t_{i} \in[0, t)} e^{\alpha\left(t-t_{i}\right)} \Delta_{i}-\sum_{t_{i} \in[0, t)} e^{\beta\left(t-t_{i}\right)} \widetilde{\Delta}_{i}\right\} .
\end{aligned}
$$

By the boundary condition of (2.1), we have

$$
\begin{aligned}
& u^{\prime}(0)-\beta u(0)=-\frac{1}{1+e^{\alpha T}}\left[\int_{0}^{T} e^{\alpha(T-s)} h(s) d s+\sum_{i=1}^{m} \Delta_{i} e^{\alpha\left(T-t_{i}\right)}\right] \\
& \alpha u(0)-u^{\prime}(0)=\frac{1}{1+e^{\beta T}}\left[\int_{0}^{T} e^{\beta(T-s)} h(s) d s+\sum_{i=1}^{m} \widetilde{\Delta}_{i} e^{\beta\left(T-t_{i}\right)}\right] .
\end{aligned}
$$


Substituting (2.21) into (2.20), and also noting that for $t \in\left(t_{k}, t_{k+1}\right]$,

$$
\begin{aligned}
-\sum_{i=1}^{m} \frac{\Delta_{i}}{1+e^{\alpha T}} e^{\alpha\left(T+t-t_{i}\right)}+\sum_{t_{i} \in[0, t]} e^{\alpha\left(t-t_{i}\right)} \Delta_{i} & =-\sum_{i=k+1}^{m} \frac{e^{\alpha\left(T+t-t_{i}\right)} \Delta_{i}}{1+e^{\alpha T}}+\sum_{i=1}^{k} \frac{e^{\alpha\left(t-t_{i}\right)} \Delta_{i}}{1+e^{\alpha T}}, \\
\sum_{i=1}^{m} \frac{\tilde{\Delta}_{i}}{1+e^{\beta T}} e^{\beta\left(T+t-t_{i}\right)}-\sum_{t_{i} \in[0, t]} e^{\beta\left(t-t_{i}\right)} \widetilde{\Delta}_{i} & =\sum_{i=k+1}^{m} \frac{e^{\alpha\left(T+t-t_{i}\right)} \widetilde{\Delta}_{i}}{1+e^{\alpha T}}-\sum_{i=1}^{k} \frac{e^{\beta\left(t-t_{i}\right)} \tilde{\Delta}_{i}}{1+e^{\beta T}}
\end{aligned}
$$

we see that $u(t)$ is the solution of (2.2).

Now assume $u(t)$ is a solution of (2.2). Then for $t \neq t_{k}, k=1,2, \ldots, m$.

$$
\begin{aligned}
& u^{\prime}(t)=\int_{0}^{T} G_{t}(t, s) h(s) d s+\sum_{i=1}^{m} H_{t}\left(t, t_{i}\right) I_{i}\left(u\left(t_{i}\right)\right)+\sum_{i=1}^{m} G_{t}\left(t, t_{i}\right) J_{i}\left(u\left(t_{i}\right)\right) \\
& u^{\prime \prime}(t)=\int_{0}^{T} G_{t t}(t, s) h(s) d s+h(t)+\sum_{i=1}^{m} H_{t t}\left(t, t_{i}\right) I_{i}\left(u\left(t_{i}\right)\right)+\sum_{i=1}^{m} G_{t t}\left(t, t_{i}\right) J_{i}\left(u\left(t_{i}\right)\right) .
\end{aligned}
$$

It is easy to verify

$$
u^{\prime \prime}(t)-p u^{\prime}(t)-q(t)=h(t)
$$

For $t=t_{k}, k=1,2, \ldots, m$, we compute straightforwardly to get

$$
\begin{gathered}
H\left(t_{k}^{+}, t_{k}\right)-H\left(t_{k}, t_{k}\right)=1, \quad G\left(t_{k}^{+}, t_{k}\right)-G\left(t_{k}, t_{k}\right)=0, \\
G_{t}\left(t_{k}^{+}, t_{k}\right)-G_{t}\left(t_{k}, t_{k}\right)=1, \quad H_{t}\left(t_{k}^{+}, t_{k}\right)-H_{t}\left(t_{k}, t_{k}\right)=0,
\end{gathered}
$$

which implies

$$
\Delta u\left(t_{k}\right)=I_{k}\left(u\left(t_{k}\right)\right), \quad \Delta u^{\prime}\left(t_{k}\right)=J_{k}\left(u\left(t_{k}\right)\right)
$$

Now, we prove $u(t)$ is a solution of (2.1). Then the proof is completed.

For later use, we present the following estimations:

$$
\begin{aligned}
& \max _{(t, s) \in[0, T] \times[0, T]}|G(t, s)| \leq \frac{1}{\alpha-\beta}\left(\frac{e^{\alpha T}}{1+e^{\alpha T}}+\frac{e^{\beta T}}{1+e^{\beta T}}\right):=G_{0}, \\
& \max _{(t, s) \in[0, T] \times[0, T]}|H(t, s)| \leq \frac{\alpha}{\alpha-\beta}\left(\frac{e^{\alpha T}}{1+e^{\alpha T}}+\frac{e^{\beta T}}{1+e^{\beta T}}\right)=\alpha G_{0}
\end{aligned}
$$




$$
\begin{gathered}
\max _{(t, s) \in[0, T] \times[0, T]}|G(t, s)| \leq \frac{\alpha}{\alpha-\beta}\left(\frac{e^{\alpha T}}{1+e^{\alpha T}}+\frac{e^{\beta T}}{1+e^{\beta T}}\right)=\alpha G_{0}, \\
\max _{(t, s) \in[0, T] \times[0, T]}\left|H_{t}(t, s)\right| \leq \frac{|\alpha| \cdot|\beta|}{\alpha-\beta}\left(\frac{e^{\alpha T}}{1+e^{\alpha T}}+\frac{e^{\beta T}}{1+e^{\beta T}}\right) \leq \alpha^{2} G_{0} .
\end{gathered}
$$

Corollary 2.2. Assume in (2.1) that $p=0$ and $q=M^{2}>0$. Then for any $h(t) \in P C[0, T], u(t)$ is the solution of

$$
\begin{gathered}
u^{\prime \prime}-M^{2} u=h(t), \quad t \in[0, T], t \neq t_{k}, k=1,2, \ldots, m, \\
u\left(t_{k}^{+}\right)=u\left(t_{k}\right)+I_{k}\left(u\left(t_{k}\right)\right), \quad u^{\prime}\left(t_{k}^{+}\right)=u^{\prime}\left(t_{k}\right)+J_{k}\left(u\left(t_{k}\right)\right), \quad k=1,2, \ldots, m, \\
u(0)=-u(T), \quad u^{\prime}(0)=-u^{\prime}(T)
\end{gathered}
$$

if and only if $u(t)$ is the solution of integral equation

$$
u(t)=\int_{0}^{T} G(t, s) h(s) d s+\sum_{i=1}^{m} H\left(t, t_{i}\right) I_{i}\left(u\left(t_{i}\right)\right)+\sum_{i=1}^{m} G\left(t, t_{i}\right) J_{i}\left(u\left(t_{i}\right)\right)
$$

where

$$
\begin{aligned}
& G(t, s)=\frac{1}{2 M} \begin{cases}-\frac{e^{M(T+t-s)}}{1+e^{M T}}+\frac{e^{-M(T+t-s)}}{1+e^{-M T}}, & 0 \leq t \leq s \leq T, \\
\frac{e^{M(t-s)}}{1+e^{M T}}-\frac{e^{-M(t-s)}}{1+e^{-M T}}, & 0 \leq s<t \leq T,\end{cases} \\
& H(t, s)=G_{t}(t, s)=\frac{1}{2 M} \begin{cases}\frac{-M e^{M(T+t-s)}}{1+e^{M T}}-\frac{M e^{-M(T+t-s)}}{1+e^{-M T}}, & 0 \leq t \leq s \leq T, \\
\frac{M e^{M(t-s)}}{1+e^{M T}}+\frac{M e^{-M(t-s)}}{1+e^{-M T}}, & 0 \leq s<t \leq T .\end{cases}
\end{aligned}
$$

Obviously, there hold

$$
\begin{array}{cl}
\max _{(t, s) \in[0, T] \times[0, T]}|G(t, s)| \leq \frac{e^{M T}}{M\left(1+e^{M T}\right)}, & \max _{(t, s) \in[0, T] \times[0, T]}|H(t, s)| \leq \frac{e^{M T}}{1+e^{M T}}, \\
\max _{(t, s) \in[0, T] \times[0, T]}\left|G_{t}(t, s)\right| \leq \frac{e^{M T}}{1+e^{M T}}, \quad & \max _{(t, s) \in[0, T] \times[0, T]}\left|H_{t}(t, s)\right| \leq \frac{M e^{M T}}{1+e^{M T}} .
\end{array}
$$

We now give Green's function of (2.1) for $p=q=0$. 
Lemma 2.3. For any $h(t) \in P C[0, T], u(t)$ is the solution of

$$
\begin{gathered}
u^{\prime \prime}=h(t), \quad t \in[0, T], \quad t \neq t_{k}, \quad k=1,2, \ldots, m, \\
u\left(t_{k}^{+}\right)=u\left(t_{k}\right)+I_{k}\left(u\left(t_{k}\right)\right), \quad u^{\prime}\left(t_{k}^{+}\right)=u^{\prime}\left(t_{k}\right)+J_{k}\left(u\left(t_{k}\right)\right), \\
u(0)=-u(T), \quad u^{\prime}(0)=-u^{\prime}(T)
\end{gathered}
$$

if and only if $u(t)$ satisfies the integral equation

$$
u(t)=\int_{0}^{T} G(t, s)^{*} h(s) d s+\sum_{i=1}^{m} H^{*}\left(t, t_{i}\right) I_{i}\left(u\left(t_{i}\right)\right)+\sum_{i=1}^{m} G^{*}\left(t, t_{i}\right) J_{i}\left(u\left(t_{i}\right)\right)
$$

where

$$
\begin{gathered}
G^{*}(t, s)=-\frac{1}{2} \begin{cases}\frac{T}{2}+t-s, & 0 \leq t \leq s \leq T, \\
\frac{T}{2}-t+s, & 0 \leq s<t \leq T,\end{cases} \\
H^{*}(t, s)=G^{*}(t, s)_{t}= \begin{cases}-\frac{1}{2}, & 0 \leq t \leq s \leq T, \\
\frac{1}{2}, & 0 \leq s<t \leq T .\end{cases}
\end{gathered}
$$

Since the proof is very similar to that of Lemma 2.1, we omit it here. We can check easily that $u(t)$ satisfies (2.34) and hence $u(t)$ is a solution of (2.33). Also we get by straightforward computation that

$$
\max _{(t, s) \in[0, T] \times[0, T]}\left|G^{*}(t, s)\right| \leq \frac{T}{4}, \quad \max _{(t, s) \in[0, T] \times[0, T]}\left|G_{t}^{*}(t, s)\right|=\max _{(t, s) \in[0, T] \times[0, T]}\left|H^{*}(t, s)\right| \leq \frac{1}{2} .
$$

Recall that a mapping between Banach spaces is compact if it is continuous and carries bounded sets into relatively compact sets.

Lemma 2.4. Suppose that $f:[0, T] \times \mathbb{R}^{n} \times \mathbb{R}^{n}$ and $I, J: \mathbb{R}^{n} \rightarrow \mathbb{R}^{n}$ are continuous. Define an operator $A: P C^{1}\left([0, T], \mathbb{R}^{n}\right) \rightarrow P C^{1}\left([0, T], \mathbb{R}^{n}\right)$ as

$$
\begin{aligned}
A u(t):= & \int_{0}^{T} G(t, s)\left(f\left(s, u(s), u^{\prime}(s)\right)-p u^{\prime}(s)-q u(s)\right) d s \\
& +\sum_{i=1}^{m} H\left(t, t_{i}\right) I_{i}\left(u\left(t_{i}\right)\right)+\sum_{i=1}^{m} G\left(t, t_{i}\right) J_{i}\left(u\left(t_{i}\right)\right),
\end{aligned}
$$

where $G(t, s)$ and $H(t, s)$ are as given in Lemma 2.1. Then $A$ is a compact map. 
Proof. Noting the continuity of $f$ and $I_{k}, J_{k}$, this follows in a standard step-by-step process and so it is omitted.

\section{Main results}

In this section, we prove the existence results for (1.1) in presence of Schaefer's fixed-point theorem.

Theorem 3.1. Suppose that $f:[0, T] \times \mathbb{R}^{n} \times \mathbb{R}^{n}$ and $I, J: \mathbb{R}^{n} \rightarrow \mathbb{R}^{n}$ are continuous. If for some $p \geq 0$ and $q>0$, there exist nonnegative constants $\gamma, \delta_{k}, \zeta_{k}, L_{k}, N_{k}$, and $M$ such that

$$
\begin{gathered}
\|f(t, x, y)-p y-q x\| \leq r\left[\langle x+y, f(t, x, y)\rangle+\|y\|^{2}\right]+M, \\
\forall(t, x, y) \in\left([0, T] \backslash \bigcup_{i=0}^{m} t_{i}\right) \times \mathbb{R}^{n} \times \mathbb{R}^{n}, \\
\left\|I_{k}(x)\right\| \leq \delta_{k}\|x\|+L_{k}, \quad\left\|J_{k}(x)\right\| \leq \zeta_{k}\|x\|+N_{k}, \quad \forall x \in \mathbb{R}^{n}, \\
\sum_{k=1}^{m} \delta_{k}+\sum_{k=1}^{m} \zeta_{k}<\frac{1}{H^{\prime}}
\end{gathered}
$$

where $\langle\cdot\rangle$ is the Euclidean inner product, $H=\max \left\{G_{0}, \alpha G_{0}, \alpha^{2} G_{0}\right\}$. Then (1.1) has at least one solution.

Proof. Define an integral operator $A$ as

$$
A u=\int_{0}^{T} G(t, s)\left(f\left(s, u(s), u^{\prime}(s)\right)-p u^{\prime}(s)-q u(s)\right) d s+\sum_{i=1}^{m} H\left(t, t_{i}\right) I_{i}\left(u\left(t_{i}\right)\right)+\sum_{i=1}^{m} G\left(t, t_{i}\right) J_{i}\left(u\left(t_{i}\right)\right),
$$

where $G(t, s)$ and $H(t, s)$ follow the forms of $(G)$ and $(H)$ in Lemma 2.1. By Lemma 2.4, $A$ is a compact mapping. Also, it follows from Lemma 2.1 that $u(t)$ is a fixed point of $A$ if and only if $u(t)$ satisfies

$$
\begin{gathered}
u^{\prime \prime}(t)-p u^{\prime}(t)-q u(t)=f\left(t, u(t), u^{\prime}(t)\right)-p u^{\prime}(t)-q u(t), \quad t \neq t_{k}, \quad k=1,2, \ldots, m, \\
u\left(t_{k}^{+}\right)=u\left(t_{k}\right)+I_{k}\left(u\left(t_{k}\right)\right), \quad u^{\prime}\left(t_{k}^{+}\right)=u^{\prime}\left(t_{k}\right)+J_{k}\left(u\left(t_{k}\right)\right), \quad k=1,2, \ldots, m, \\
u(0)=-u(T), \quad u^{\prime}(0)=-u^{\prime}(T),
\end{gathered}
$$

which is equivalent to (1.1). Consequently, all that we need to do is to verify that $A$ has at least one fixed point. With this in mind, we assume $u(t)$ is a solution of

$$
u=\lambda A u, \quad \lambda \in(0,1) .
$$


That is,

$$
\begin{aligned}
u(t)= & \int_{0}^{T} G(t, s) \lambda\left[f\left(s, u(s), u^{\prime}(s)\right)-p u^{\prime}(s)-q u(s)\right] d s \\
& +\lambda \sum_{i=1}^{m} H\left(t, t_{i}\right) I_{i}\left(u\left(t_{i}\right)\right)+\lambda \sum_{i=1}^{m} G\left(t, t_{i}\right) J_{i}\left(u\left(t_{i}\right)\right) .
\end{aligned}
$$

It is equivalent to say that $u(t)$ satisfies

$$
\begin{gathered}
u^{\prime \prime}(t)-p u^{\prime}(t)-q u(t)=\lambda\left[f\left(t, u(t), u^{\prime}(t)\right)-p u^{\prime}(t)-q u(t)\right], \quad t \neq t_{k}, \quad k=1,2, \ldots, m, \\
u\left(t_{k}^{+}\right)=u\left(t_{k}\right)+\lambda I_{k}\left(u\left(t_{k}\right)\right), \quad u^{\prime}\left(t_{k}^{+}\right)=u^{\prime}\left(t_{k}\right)+\lambda J_{k}\left(u\left(t_{k}\right)\right), \quad k=1,2, \ldots, m, \\
u(0)=-u(T), \quad u^{\prime}(0)=-u^{\prime}(T) .
\end{gathered}
$$

Firstly, we see that for $\lambda \in(0,1)$,

$$
\begin{aligned}
& \lambda\left\|f\left(t, u(t), u^{\prime}(t)\right)-p u^{\prime}(t)-q u(t)\right\| \\
& \leq \lambda\left\{r\left[\left\langle u(t)+u^{\prime}(t), f\left(t, u(t), u^{\prime}(t)\right)\right\rangle+\left\|u^{\prime}(t)\right\|^{2}\right]+M\right\} \\
&= r\left[\left\langle u(t)+u^{\prime}(t), \lambda f\left(t, u(t), u^{\prime}(t)\right)\right\rangle+\lambda\left\|u^{\prime}(t)\right\|^{2}\right]+\lambda M \\
&= r\left[\left\langle u(t)+u^{\prime}(t), u^{\prime \prime}(t)-(1-\lambda)\left(p u^{\prime}(t)+q u(t)\right)\right\rangle+\lambda\left\|u^{\prime}(t)\right\|^{2}\right]+\lambda M \\
&= r\left[\left\langle u(t)+u^{\prime}(t), u^{\prime \prime}(t)\right\rangle-(1-\lambda)(p+q)\left\langle u(t), u^{\prime}(t)\right\rangle\right. \\
&\left.\quad-q(1-\lambda)\|u(t)\|^{2}-p(1-\lambda)\left\|u^{\prime}(t)\right\|^{2}+\lambda\left\|u^{\prime}(t)\right\|^{2}\right]+\lambda M \\
& \leq r\left[\left\langle u(t)+u^{\prime}(t), u^{\prime \prime}(t)\right\rangle-(1-\lambda)(p+q)\left\langle u(t), u^{\prime}(t)\right\rangle+\left\|u^{\prime}(t)\right\|^{2}\right]+M \\
&= r\left[\left\langle u(t)+u^{\prime}(t), u^{\prime}(t)+u^{\prime \prime}(t)\right\rangle-(1-\lambda)(p+q)\left\langle u(t), u^{\prime}(t)\right\rangle-\left\langle u(t), u^{\prime}(t)\right\rangle\right]+M .
\end{aligned}
$$

Further more, by the antiperiodic boundary condition we have

$$
\begin{gathered}
\int_{0}^{T}\left\langle u(t), u^{\prime}(t)\right\rangle d t=\frac{1}{2} \int_{0}^{T} \frac{d}{d t}\left(\|u(t)\|^{2}\right)=\frac{1}{2}\left(\|u(T)\|^{2}-\|u(0)\|^{2}\right)=0 \\
\int_{0}^{T}\left\langle u(t)+u^{\prime}(t), u^{\prime}(t)+u^{\prime \prime}(t)\right\rangle d t=\frac{1}{2}\left(\left\|u(T)+u^{\prime}(T)\right\|^{2}-\left\|u(0)+u^{\prime}(0)\right\|^{2}\right)=0 .
\end{gathered}
$$


As a result,

$$
\left.\int_{0}^{T} \lambda \| f\left(t, u(t), u^{\prime}(t)\right)-p u^{\prime}(t)-q u(t)\right) \| d t \leq M T
$$

Now we show that any potential solution of (3.6) is bounded a priori. By (3.2) and (3.11), we obtain

$$
\begin{aligned}
\|u(t)\|= & \lambda\|A u(t)\| \\
= & \| \int_{0}^{T} G(t, s) \lambda\left[f\left(t, u(t), u^{\prime}(t)\right)-p u^{\prime}(t)-q u(t)\right] d t \\
& +\lambda \sum_{i=1}^{m} H\left(t, t_{i}\right) I_{i}\left(u\left(t_{i}\right)\right)+\lambda \sum_{i=1}^{m} G\left(t, t_{i}\right) J_{i}\left(u\left(t_{i}\right)\right) \| \\
\leq & G_{0} \int_{0}^{T} \lambda\left\|f\left(t, u(t), u^{\prime}(t)\right)-p u^{\prime}(t)-q u(t)\right\| d t+\lambda \alpha G_{0} \sum_{i=1}^{m}\left\|I_{i}\left(u\left(t_{i}\right)\right)\right\|+\lambda G_{0} \sum_{i=1}^{m}\left\|J_{i}\left(u\left(t_{i}\right)\right)\right\| \\
\leq & G_{0}\left(M T+\alpha \sum_{k=1}^{m} L_{k}+\sum_{k=1}^{m} N_{k}\right)+G_{0}\left(\alpha \sum_{i=1}^{m} \zeta_{i}\left\|\left(u\left(t_{i}\right)\right)\right\|+\sum_{i=1}^{m} \delta_{i}\left\|\left(u\left(t_{i}\right)\right)\right\|\right) \\
\leq & G_{1}\left(M T+\sum_{k=1}^{m} L_{k}+\sum_{k=1}^{m} N_{k}\right)+G_{1}\left(\sum_{i=1}^{m} \zeta_{i}\left\|\left(u\left(t_{i}\right)\right)\right\|+\sum_{i=1}^{m} \delta_{i}\left\|\left(u\left(t_{i}\right)\right)\right\|\right) .
\end{aligned}
$$

Taking the supremum and rearranging, we get by (3.3) that

$$
\sup _{t \in[0, T]}\|u(t)\| \leq \frac{G_{1}\left(T M+\sum_{k=1}^{m} L_{k}+\sum_{k=1}^{m} N_{k}\right)}{1-G_{1}\left(\sum_{k=1}^{m} \delta_{k}+\sum_{k=1}^{m} \zeta_{k}\right)} .
$$

Differentiating both sides of (3.7) and noting (2.23), we obtain

$$
\sup _{t \in[0, T]}\left\|u^{\prime}(t)\right\| \leq \frac{G_{2}\left(T M+\sum_{k=1}^{m} L_{k}+\sum_{k=1}^{m} N_{k}\right)}{1-G_{2}\left(\sum_{k=1}^{m} \delta_{k}+\sum_{k=1}^{m} \zeta_{k}\right)}
$$

where

$$
G_{2}=\max \left\{\alpha G_{0}, \alpha^{2} G_{0}\right\}
$$


Thus,

$$
\|u(t)\|_{P C^{1}}=\max \left\{\frac{G_{1}\left(T M+\sum_{k=1}^{m} L_{k}+\sum_{k=1}^{m} N_{k}\right)}{1-G_{1}\left(\sum_{k=1}^{m} \delta_{k}+\sum_{k=1}^{m} \zeta_{k}\right)}, \frac{G_{2}\left(T M+\sum_{k=1}^{m} L_{k}+\sum_{k=1}^{m} N_{k}\right)}{1-G_{2}\left(\sum_{k=1}^{m} \delta_{k}+\sum_{k=1}^{m} \zeta_{k}\right)}\right\}:=R .
$$

Now we have shown that any possible solution of (3.6) is bounded by $R$ which is independent of $\lambda$. By Scheafer's fixed theorem we know that $A$ has at least one fixed point. Therefore, the proof is completed.

Suppose both $p=0$ and $q=M^{2}$ in Theorem 3.1. We obtain the following theorem.

Theorem 3.2. Assume that $f:[0, T] \times \mathbb{R}^{n} \times \mathbb{R}^{n}$ and $I, J: \mathbb{R}^{n} \rightarrow \mathbb{R}^{n}$ are continuous. If for some $M>0$ there exist nonnegative constants $\gamma, \delta_{k}, \zeta_{k}, L_{k}, N_{k}$, and $M^{*}$ such that

$$
\begin{gathered}
\left\|f(t, x, y)-M^{2} x\right\| \leq \gamma\left[\langle x, f(t, x, y)\rangle+\|y\|^{2}\right]+M^{*}, \\
\forall(t, x, y) \in\left([0, T] \backslash \bigcup_{i=0}^{m} t_{i}\right) \times \mathbb{R}^{n} \times \mathbb{R}^{n}, \\
\left\|I_{k}(x)\right\| \leq \delta_{k}\|x\|+L_{k}, \quad\left\|J_{k}(x)\right\| \leq \zeta_{k}\|x\|+N_{k}, \quad \forall x \in \mathbb{R}^{n}, \\
\sum_{k=1}^{m} \delta_{k}+\sum_{k=1}^{m} \zeta_{k}<\frac{1}{\check{H}^{\prime}}
\end{gathered}
$$

where $\langle\cdot\rangle$ is the Euclidean inner product, $\check{H}=\max \left\{e^{M T} / M\left(1+e^{M T}\right), e^{M T} /\left(1+e^{M T}\right), M e^{M T} /\right.$ $\left.M\left(1+e^{M T}\right)\right\}$, then (1.1) has at least one solution.

Proof. Consider the mapping

$$
\begin{gathered}
A: P C^{1}\left([0, T], \mathbb{R}^{n}\right) \longrightarrow P C^{1}\left([0, T], \mathbb{R}^{n}\right), \\
A u(t)=\int_{0}^{T} G(t, s) h(s) d s+\sum_{i=1}^{m} H\left(t, t_{i}\right) I_{i}\left(u\left(t_{i}\right)\right)+\sum_{i=1}^{m} G\left(t, t_{i}\right) J_{i}\left(u\left(t_{i}\right)\right),
\end{gathered}
$$

where

$$
\begin{aligned}
& G(t, s)=\frac{1}{2 M} \begin{cases}-\frac{e^{M(T+t-s)}}{1+e^{M T}}+\frac{e^{-M(T+t-s)}}{1+e^{-M T}}, & 0 \leq t \leq s \leq T, \\
\frac{e^{M(t-s)}}{1+e^{M T}}-\frac{e^{-M(t-s)}}{1+e^{-M T}}, & 0 \leq s<t \leq T,\end{cases} \\
& H(t, s)=G_{t}(t, s)=\frac{1}{2 M} \begin{cases}\frac{-M e^{M(T+t-s)}}{1+e^{M T}}-\frac{M e^{-M(T+t-s)}}{1+e^{-M T}}, & 0 \leq t \leq s \leq T, \\
\frac{M e^{M(t-s)}}{1+e^{M T}}+\frac{M e^{-M(t-s)}}{1+e^{-M T}}, & 0 \leq s<t \leq T .\end{cases}
\end{aligned}
$$


By Lemma 2.4, $A$ is a compact mapping. Consider the equation

$$
u=A u .
$$

To show that $A$ has at least one fixed point, we apply Schaefer's theorem by showing that all potential solutions to

$$
u=\lambda A u, \quad \lambda \in(0,1),
$$

are bounded a priori, with the bound being independent of $\lambda$. With this in mind, let $u(t)$ be a solution of (3.23). Note that $u(t)$ is also a solution to

$$
\begin{gathered}
u^{\prime \prime}(t)-M^{2} u(t)=\lambda\left[f\left(t, u(t), u^{\prime}(t)\right)-M^{2} u(t)\right], \quad t \neq t_{k}, \quad k=1,2, \ldots, m, \\
u\left(t_{k}^{+}\right)=u\left(t_{k}\right)+\lambda I_{k}\left(u\left(t_{k}\right)\right), \quad u^{\prime}\left(t_{k}^{+}\right)=u^{\prime}\left(t_{k}\right)+\lambda J_{k}\left(u\left(t_{k}\right)\right), \quad k=1,2, \ldots, m, \\
u(0)=-u(T), \quad u^{\prime}(0)=-u^{\prime}(T) .
\end{gathered}
$$

On one hand, we see that for $\lambda \in(0,1)$,

$$
\begin{aligned}
\lambda\left\|f\left(t, u(t), u^{\prime}(t)\right)\right\| \leq \lambda\left\{r\left[\left\langle u(t), f\left(t, u(t), u^{\prime}(t)\right)\right\rangle+\left\|u^{\prime}(t)\right\|^{2}\right]+M^{*}\right\} \\
\quad=r\left[\left\langle u(t), \lambda f\left(t, u(t), u^{\prime}(t)\right)\right\rangle+\lambda\left\|u^{\prime}(t)\right\|^{2}\right]+\lambda M \\
\left.\quad=r\left[\left\langle u(t), u^{\prime \prime}(t)-(1-\lambda) M^{2} u(t)\right)\right\rangle+\lambda\left\langle u^{\prime}(t), u^{\prime}(t)\right\rangle\right]+\lambda M^{*} \\
\quad=r\left[\left\langle u(t), u^{\prime \prime}(t)\right\rangle-(1-\lambda) M^{2}\|u(t)\|^{2}+\lambda\left\langle u^{\prime}(t), u^{\prime}(t)\right\rangle\right]+\lambda M^{*} \\
\quad \leq r\left[\left\langle u(t), u^{\prime \prime}\right\rangle+\left\langle u^{\prime}(t), u^{\prime}(t)\right\rangle\right]+M^{*} \\
\quad=r \frac{d}{d t}\left\langle u(t), u^{\prime}(t)\right\rangle+M^{*} .
\end{aligned}
$$

On the other hand, by the antiperiodic boundary condition we have

$$
\int_{0}^{T}\left[\left\langle u(t), u^{\prime \prime}\right\rangle+\left\langle u^{\prime}(t), u^{\prime}(t)\right\rangle\right] d t=\left\langle u(T), u^{\prime}(T)\right\rangle-\left\langle u(0), u^{\prime}(0)\right\rangle=\left\langle-u(0),-u^{\prime}(0)\right\rangle-\left\langle u(0), u^{\prime}(0)\right\rangle=0 .
$$


It therefore follows that

$$
\int_{0}^{T} \lambda\left\|f\left(t, u(t), u^{\prime}(t)\right)\right\| d t \leq M^{*} T
$$

Consequently,

$$
\begin{aligned}
\|u(t)\| & =\lambda\|A u(t)\| \\
& =\left\|\int_{0}^{T} G(t, s) \lambda\left[f\left(t, u(t), u^{\prime}(t)\right)\right] d t+\lambda \sum_{i=1}^{m} H\left(t, t_{i}\right) I_{i}\left(u\left(t_{i}\right)\right)+\lambda \sum_{i=1}^{m}\left(t, t_{i}\right) J_{i}\left(u\left(t_{i}\right)\right)\right\| \\
& \leq \breve{G}_{0} \int_{0}^{T} \lambda\left\|f\left(t, u(t), u^{\prime}(t)\right)-p u^{\prime}(t)-q u(t)\right\| d t+\lambda M \breve{G}_{0} \sum_{i=1}^{m}\left\|I_{i}\left(u\left(t_{i}\right)\right)\right\|+\lambda \check{G}_{0} \sum_{i=1}^{m}\left\|\left(u\left(t_{i}\right)\right)\right\| \\
& \leq \check{G}_{1}\left(M^{*} T+\sum_{k=1}^{m} L_{k}+\sum_{k=1}^{m} N_{k}\right)+\check{G}_{1}\left(\sum_{i=1}^{m} \zeta_{i}\left\|\left(u\left(t_{i}\right)\right)\right\|+\sum_{i=1}^{m} \delta_{i}\left\|\left(u\left(t_{i}\right)\right)\right\|\right),
\end{aligned}
$$

where $\breve{G}_{0}=e^{M T} / M\left(1+e^{M T}\right), \check{G}_{1}=\max \left\{e^{M T} / M\left(1+e^{M T}\right), e^{M T} /\left(1+e^{M T}\right)\right\}$.

We compute directly to get

$$
\sup _{t \in[0, T]}\|u(t)\| \leq \frac{\check{G}_{1}\left(T M^{*}+\sum_{k=1}^{m} L_{k}+\sum_{k=1}^{m} N_{k}\right)}{1-\breve{G}_{1}\left(\sum_{k=1}^{m} \delta_{k}+\sum_{k=1}^{m} \zeta_{k}\right)} .
$$

Differentiating both sides of (3.19), we obtain

$$
\sup _{t \in[0, T]}\left\|u^{\prime}(t)\right\| \leq \frac{\check{G}_{2}\left(T^{*} M+\sum_{k=1}^{m} L_{k}+\sum_{k=1}^{m} N_{k}\right)}{1-\check{G}_{2}\left(\sum_{k=1}^{m} \delta_{k}+\sum_{k=1}^{m} \zeta_{k}\right)}
$$

where

$$
\check{G}_{2}=\max \left\{\frac{e^{M T}}{1+e^{M T}}, \frac{M e^{M T}}{1+e^{M T}}\right\} .
$$

Thus,

$$
\|u(t)\|_{P C^{1}}=\max \left\{\frac{\breve{G}_{1}\left(T M^{*}+\sum_{k=1}^{m} L_{k}+\sum_{k=1}^{m} N_{k}\right)}{1-\breve{G}_{1}\left(\sum_{k=1}^{m} \delta_{k}+\sum_{k=1}^{m} \zeta_{k}\right)}, \frac{\breve{G}_{2}\left(T M^{*}+\sum_{k=1}^{m} L_{k}+\sum_{k=1}^{m} N_{k}\right)}{1-\breve{G}_{2}\left(\sum_{k=1}^{m} \delta_{k}+\sum_{k=1}^{m} \zeta_{k}\right)}\right\}:=\check{R} .
$$

Then the proof is completed. 
Similarly, we can prove the following existence result for $M=0$ in Theorem 3.2.

Theorem 3.3. Suppose that $f:[0, T] \times \mathbb{R}^{n} \times \mathbb{R}^{n}$ and $I, J: \mathbb{R}^{n} \rightarrow \mathbb{R}^{n}$ are continuous. If there exist nonnegative constants $\gamma, \delta_{k}, \zeta_{k}, L_{k}, N_{k}$, and $M$ such that

$$
\begin{gathered}
\|f(t, x, y)\| \leq r\left[\langle x, f(t, x, y)\rangle+\|y\|^{2}\right]+M, \quad(t, x, y) \in\left([0, T] \backslash \bigcup_{i=0}^{m} t_{i}\right) \times \mathbb{R}^{n} \times \mathbb{R}^{n}, \\
\left\|I_{k}(x)\right\| \leq \delta_{k}\|x\|+L_{k}, \quad\left\|J_{k}(x)\right\| \leq \zeta_{k}\|x\|+N_{k}, \quad \forall x \in \mathbb{R}^{n}, \\
\sum_{k=1}^{m} \delta_{k}+\sum_{k=1}^{m} \zeta_{k}<\frac{1}{G_{0}^{*}},
\end{gathered}
$$

where $\langle\cdot\rangle$ is the Euclidean inner product, $G_{0}^{*}=\max \{T / 4,1 / 2\}$, then (1.1) has at least one solution.

\section{Examples}

In this part, we show how our main theorems work by a couple of examples.

Example 4.1. The scalar second-order impulsive equations with antiperiodic boundary value condition

$$
\begin{gathered}
u^{\prime \prime}=\left(u(t)+u^{\prime}(t)\right)^{3}+2 u(t)+u^{\prime}(t)+t, \quad t \in[0,1], t \neq t_{1}, \\
u\left(t_{1}^{+}\right)=u\left(t_{1}\right)+\frac{1}{10} u\left(t_{1}\right)+1, \quad u^{\prime}\left(t_{1}^{+}\right)=u^{\prime}\left(t_{1}\right)-\frac{1}{10} u\left(t_{1}\right)+2, \\
u(0)=-u(1), \quad u^{\prime}(0)=-u^{\prime}(1),
\end{gathered}
$$

where $t_{1} \in(0,1)$, have at least one solution.

Proof. Let $T=1$ and $f(t, x, y)=(x+y)^{3}+2 x+y+t$ in Theorem 3.1. For $p=1, q=2$, we have $\alpha=2, \beta=-1$, and

$$
|f(t, x, y)-y-2 x|=|x+y|^{3}+1, \quad \forall(t, x, y) \in[0,1] \times \mathbb{R}^{2}
$$

On the other hand, for $(t, x, y) \in[0,1] \times \mathbb{R}^{2}$,

$$
\begin{aligned}
\langle(x+y), f(t, x, y)\rangle+y^{2} & =(x+y)^{4}+2 x^{2}+3 x y+2 y^{2}+(x+y) t \\
& \geq(x+y)^{4}-|x+y|+4|x| \cdot|y|+3 x y \\
& \geq(x+y)^{4}-|x+y| .
\end{aligned}
$$


Noting $\min _{v \geq 0}\left\{v^{4}-v^{3}-v\right\}>-2$, we have for $\gamma=1$ and $M=3$ that

$$
\gamma\left[\langle(x+y), f(t, x, y)\rangle+y^{2}\right]+M \geq|f(t, x, y)-y-2 x|, \quad \forall(t, x, y) \in[0,1] \times \mathbb{R}^{2} .
$$

Moreover, $H=\alpha^{2} G_{0}=(4 / 3)\left(e^{2} /\left(1+e^{2}\right)+e^{-1} /\left(1+e^{-1}\right)\right) \approx 1.53298, \delta_{1}+\zeta_{1}=0.2<1 / H$. Then the conclusion follows from Theorem 3.1.

Example 4.2. Consider antiperiodic value problem

$$
\begin{gathered}
u^{\prime \prime}(t)=u(t)+u(t) u^{\prime}(t)^{2}+\cos t, \quad t \in[0,1], t \neq t_{1}, \\
u\left(t_{1}^{+}\right)=u\left(t_{1}\right)+\frac{1}{4} u\left(t_{1}\right)+4, \quad u^{\prime}\left(t_{1}^{+}\right)=u^{\prime}\left(t_{1}\right)-\frac{1}{2} u\left(t_{1}\right), \\
u(0)=-u(1), \quad u^{\prime}(0)=-u^{\prime}(1) .
\end{gathered}
$$

We claim that (4.5) has at least one solution.

Proof. Let $T=1$ and $f(t, x, y)=x+x y^{2}+\cos t$ in Theorem 3.2. Choosing $M=1$, we have for $(t, x, y) \in[0,1] \times \mathbb{R}^{2}$ that

$$
\begin{gathered}
|f(t, x, y)-x|=|x| y^{2}+\cos t \\
\langle x, f(t, x, y)\rangle+y^{2}=x^{2}+x^{2} y^{2}+y^{2}+x \cos t \geq x^{2}+x^{2} y^{2}+y^{2}-|x| .
\end{gathered}
$$

Since $\min _{v \geq 0}\left\{v^{2}-v\right\}>-1$, we have $x^{2} y^{2}+y^{2}-|x| y^{2}=y^{2}\left(x^{2}-|x|+1\right)>0$. Thus, for $r=1$ and $M^{*}=2$,

$$
\gamma\left[\langle x, f(t, x, y)\rangle+y^{2}\right]+M^{*} \geq|f(t, x, y)-x|, \quad \forall(t, x, y) \in[0,1] \times \mathbb{R}^{2} .
$$

Moreover, $\check{H}=e /(1+e), \delta_{1}+\zeta_{1}=3 / 4<1 / \check{H}$. Then the conclusion follows from Theorem 3.2.

\section{Acknowledgments}

This research is supported by Ad Futura Scientific and Educational Foundation of the Republic of Slovenia, the Ministry of Higher Education, Science and Technology of the Republic of Slovenia; the Nova Kreditna Banka Maribor; TELEKOM Slovenije; National Natural Science Foundation of China (10671127); National Natural Science Foundation of Shanghai (08ZR1416000); and Foundation of Science and Technology Commission of Shanghai Municipality (06XD14034).

\section{References}

[1] D. BaĬnov and P. S. Simeonov, Impulsive Differential Equations: Periodic Solutions and Applications, vol. 66 of Pitman Monographs and Surveys in Pure and Applied Mathematics, Longman Scientific \& Technical, Harlow, UK, 1993.

[2] M. Benchohra, J. Henderson, and S. Ntouyas, Impulsive Differential Equations and Inclusions, vol. 2 of Contemporary Mathematics and Its Applications, Hindawi, New York, NY, USA, 2006. 
[3] D. Franco and J. J. Nieto, "First-order impulsive ordinary differential equations with anti-periodic and nonlinear boundary conditions," Nonlinear Analysis: Theory, Methods $\mathcal{E}$ Applications, vol. 42, no. 2, pp. 163-173, 2000.

[4] J. Y. Park and T. G. Ha, "Existence of antiperiodic solutions for hemivariational inequalities," Nonlinear Analysis: Theory, Methods \& Applications, vol. 68, no. 4, pp. 747-767, 2008.

[5] T. Jankowski, "Ordinary differential equations with nonlinear boundary conditions of antiperiodic type," Computers \& Mathematics with Applications, vol. 47, no. 8-9, pp. 1419-1428, 2004.

[6] A. R. Aftabizadeh, S. Aizicovici, and N. H. Pavel, "Anti-periodic boundary value problems for higher order differential equations in Hilbert spaces," Nonlinear Analysis: Theory, Methods E Applications, vol. 18, no. 3, pp. 253-267, 1992.

[7] S. Aizicovici, M. McKibben, and S. Reich, "Anti-periodic solutions to nonmonotone evolution equations with discontinuous nonlinearities," Nonlinear Analysis: Theory, Methods E Applications, vol. 43, no. 2, pp. 233-251, 2001.

[8] S. Aizicovici and N. H. Pavel, "Anti-periodic solutions to a class of nonlinear differential equations in Hilbert space," Journal of Functional Analysis, vol. 99, no. 2, pp. 387-408, 1991.

[9] A. Cabada and D. R. Vivero, "Existence and uniqueness of solutions of higher-order antiperiodic dynamic equations," Advances in Difference Equations, vol. 2004, no. 4, pp. 291-310, 2004.

[10] K. Wang, "A new existence result for nonlinear first-order anti-periodic boundary value problems," Applied Mathematics Letters, vol. 21, no. 11, pp. 1149-1154, 2008.

[11] K. Wang and Y. Li, "A note on existence of (anti-)periodic and heteroclinic solutions for a class of second-order odes," Nonlinear Analysis: Theory, Methods \& Applications., vol. 70, no. 4, pp. 1711-1724, 2009.

[12] W. Wang and J. Shen, "Existence of solutions for anti-periodic boundary value problems," Nonlinear Analysis: Theory, Methods \& Applications, vol. 70, no. 2, pp. 598-605, 2009.

[13] J. J. Nieto and C. C. Tisdell, "Existence and uniqueness of solutions to first-order systems of nonlinear impulsive boundary-value problems with sub-, super-linear or linear growth," Electronic Journal of Differential Equations, vol. 2007, no. 105, pp. 1-14, 2007.

[14] Y. Chen, J. J. Nieto, and D. O’Regan, "Anti-periodic solutions for fully nonlinear first-order differential equations," Mathematical and Computer Modelling, vol. 46, no. 9-10, pp. 1183-1190, 2007.

[15] W. Ding, Y. Xing, and M. Han, "Anti-periodic boundary value problems for first order impulsive functional differential equations," Applied Mathematics and Computation, vol. 186, no. 1, pp. 45-53, 2007.

[16] Z. Luo, J. Shen, and J. J. Nieto, "Antiperiodic boundary value problem for first-order impulsive ordinary differential equations," Computers $\mathcal{E}$ Mathematics with Applications, vol. 49, no. 2-3, pp. 253$261,2005$.

[17] A. Cabada, E. Liz, and S. Lois, "Green's function and maximum principle for higher order ordinary differential equations with impulses," The Rocky Mountain Journal of Mathematics, vol. 30, no. 2, pp. 435-446, 2000.

[18] J. Chen, C. C. Tisdell, and R. Yuan, "On the solvability of periodic boundary value problems with impulse," Journal of Mathematical Analysis and Applications, vol. 331, no. 2, pp. 902-912, 2007.

[19] M. Rudd and C. C. Tisdell, "On the solvability of two-point, second-order boundary value problems," Applied Mathematics Letters, vol. 20, no. 7, pp. 824-828, 2007. 